\title{
Diagnostic Accuracy of Anthropometric Indices for Discriminating Elevated Blood Pressure in Pediatric Population: A Systematic Review and a Meta- Analysis
}

Jun-Min Tao

Dalian Medical University

Wei Wei

Dalian Municipal Central Hospital

Xiao-Yang Ma

Dalian Medical University

Ying-Xiang Huo

Dalian Medical University

Meng-Die Hu

Dalian Medical University

Xiao-Feng Li

Dalian Medical University

Xin Chen ( $\square$ chenx@dmu.edu.cn )

Dalian Medical University

Research Article

Keywords: Body mass index, waist circumference, waist-to-height ratio, elevated blood pressure, children and adolescents

Posted Date: August 11th, 2021

DOI: https://doi.org/10.21203/rs.3.rs-727288/v1

License: (c) (i) This work is licensed under a Creative Commons Attribution 4.0 International License. Read Full License

Version of Record: A version of this preprint was published at BMC Pediatrics on January 4th, 2022. See the published version at https://doi.org/10.1186/s12887-021-03062-8. 


\section{Abstract}

Background: Childhood obesity is more likely to increase the chance of many adult health problems. Numerous studies have shown obese children to be more prone to elevated blood pressure (BP) and hypertension. It is important to identify an obesity anthropometric index with good discriminatory power for them in pediatric population.

Methods: MEDLINE/PubMed, Web of Science, and Cochrane databases were retrieved comprehensively for eligible studies on childhood obesity and hypertension/elevated BP through June 2021. The systematic review and meta-analysis of studies used receiver operating characteristics (ROC) curves for evaluating the discriminatory power of body mass index (BMI), waist circumference (WC), and waist-to-height ratio (WHtR) in distinguishing children with elevated BP and hypertension.

Results: 21 cross-sectional studies involving 177,943 children and 3-19 years of age were included in our study. Meta-analysis showed that the pooled area under the reporting receiver-operating characteristic curves (AUC) and $95 \%$ confidence intervals (Cls) for BMI, WC, and WHtR to detect hypertension of boys were $0.68(0.64,0.72), 0.69(0.64,0.74), 0.67(0.63,0.71)$, for elevated BP, the pooled AUCs and 95\% Cls were $0.67(0.61,0.73), 0.65(0.58,0.73), 0.65(0.61$, 0.71). The pooled AUCs and $95 \%$ Cls for BMI, WC and WHtR of predicting hypertension were $0.70(0.66,0.75), 0.69(0.64,0.75), 0.67(0.63,0.72)$ in girls, the pooled AUCs and 95\% Cls of predicting elevated BP were $0.63(0.61,0.65), 0.62(0.60,0.65), 0.62(0.60,0.64)$ respectively. There was no anthropometric index was statistically superior in identifying hypertension and elevated BP, however, the accuracy of BMI predicting hypertension was significantly higher than elevated $\mathrm{BP}$ in girls $(P<0.05)$. The subgroup analysis for the comparison of $\mathrm{BMI}$, WC and WHtR was performed, no significant difference in predicting hypertension and elevated BP in pediatric population.

Conclusions: This systematic review showed that no anthropometric index was superior in identifying hypertension and elevated BP in pediatric population. while compared with predicting elevated BP, all the indicators showed superiority in predicting hypertension in children, the difference was especially obvious in girls. A better anthropometric index should be explored to predict children's early blood pressure abnormalities.

\section{Background}

Hypertension, as a vital cardiovascular risk factor, is estimated account for approximately $50 \%$ of the coronary heart disease burden and $67 \%$ of the cerebrovascular disease burden [1]. Hypertension is being increasingly reported in children and adolescents. Children with high blood pressure (BP) have early vascular aging and are more likely to progress to hypertension as adults [2-7]. Numerous studies have shown children with obesity to be more prone to hypertension and elevated BP [8]. Since the 1990s, the prevalence of children who are overweight and obese has increased dramatically [9]. Although the increased rate of childhood obesity in some developed countries has plateaued, the prevalence is still high [10]. Childhood obesity is more likely to lead to adulthood obesity, which can increase the chance of many adult health risk, such as heart disease, hypertension and type 2 diabetes [11]. To overcome this crucial health problem, it will be important to identify an anthropometric index with good discriminatory power that is simple to measure and interpret.

Currently, weight status can be assessed by several anthropometric indexes, such as body mass index (BMI), waist circumference (WC), and the waist-toheight ratio (WHtR); however, which index better predicts hypertension is unknown. A meta-analysis suggested that WC and the WHtR are not superior to BMI in identifying elevated BP in children, but only 9 articles were included in the previous study, of which only one Asian study was from India [12]. Therefore, the research results may have some limitations. The number of relevant studies has increased substantially. Therefore, we conducted a full robust systematic review to determine which anthropometric indices should be recommended for screening purposes.

\section{Methods}

This systematic review was conducted according to the meta-analysis of observational studies in epidemiology (MOOSE) criteria [13].

\section{Literature search strategy}

We comprehensively retrieved the MEDLINE/PubMed, Web of Science, and Cochrane databases for eligible studies involving childhood obesity and hypertension. The relevant studies were published until June 2021 were included. In addition, the key words used in the search included "body mass index or BMI," "waist circumference or WC," "waist-to-height ratio or WHtR," "elevated blood pressure or hypertension," "children," and "adolescent." All of the reference lists of included studies were also manually retrieved so that no studies were overlooked. Two independent reviewers evaluated all relevant articles and disagreements were resolved by discussion. Only English articles were included in our research.

\section{Study inclusion/exclusion criteria}

- Studies was limited to cross-sectional design;

- Studies must focus on children or adolescents, any ethnic group, age $\leq 19$ years;

- Studies assessing the association between anthropometric indices and pediatric hypertension or elevated BP;

- BMI and WC or the WHtR, measured at least one metric;

- Studies reporting receiver-operating characteristic (ROC) curve analyses with the area under the ROC curve (AUC) provided.

- All anthropometric indices in the study must be measured twice at least with the mean value recorded;

- Non- original article, such as reviews and letters, were not considered;

- Studies that involved adults only or failed to provide AUC values were excluded; 
- Non-English articles were excluded.

\section{Main anthropometric indices and measures}

WC was measured to the nearest $0.1 \mathrm{~cm}$ by a non-elastic flexible tape in the standing position. The tape was applied horizontally midway between the lowest rib margin and the iliac crest [14]. BMI was calculated based on the weight divided by height squared $\left(\mathrm{kg} / \mathrm{m}^{2}\right)$. The WHtR was calculated by dividing the WC $(\mathrm{cm})$ by height $(\mathrm{cm})$. The blood pressure was measured at least two times in each subject and the average value was obtained. The criterion of hypertension was according to the Fourth Report on the Diagnosis, Evaluation, and Treatment of High Blood Pressure in Children and Adolescents (NHBPEP) [15] and other local criteria of hypertension have also been adopted. Elevated BP was defined as the average systolic BP or diastolic BP $\geq 90$ th or $\geq 95$ th percentile for gender, age, and height.

\section{Data extraction and quality assessments}

Two reviewers independently extracted the following study characteristics from the included studies using a standard data extraction form: Study, study year, country, region, study design, characteristics, BP measurement device, sample number, age, hypertension criterion and anthropometric indices. The AUC with a 95\% confidence interval $(\mathrm{Cl})$ or standard error was extracted. The methodologic quality of included study was evaluated by the Quality Assessment of Diagnostic Accuracy Studies-2 (QUADAS-2) tool [16].

\section{Statistical analysis}

ROC analysis is a widely-used method to examine the discrimination power of anthropometric indices, because of its powerful efficiency and clear interpretation [17-20]. The AUC prefers a comprehensive summary of test performance. Perfect tests have a mean AUC close to 1, whereas poor tests have an AUC close to 0.5. Data on the mean AUC with $95 \% \mathrm{Cl}$ and sample size for each study were inputted into a database. The quality assessment of included studies was carried out by Review Manager version 5.3 (Cochrane Collaboration, Oxford, England), meta-analysis was performed using STATA version 15.3 (Stata Corp., College Station, TX, USA). If an anthropometric index had more than one type of transformation in one study, the z-score was prior because the zscore is a standardized value. The heterogeneity of the studies was measured using the $P$ statistic. When an $P$ was $>50 \%$ or a $P$ value was $<0.05$ was identified for heterogeneity among studies, we used the random-effects model, otherwise, a fixed-effects model was adopted. To further analyze the differences between BMI, WC and WHtR, subgroup analysis was used separately for region, hypertension criterion, hypertension diagnostic basis and BP measurement device. The regions of studies were classified according to continent (Europe, America and Asia); the hypertension and elevated blood pressure criterion contained average systolic BP or diastolic BP $\geq 90$ th and average systolic BP or diastolic BP $\geq 95$ th percentile; diagnostic basis included NHBPEP and other local criteria of hypertension. BP measurement device was divided into mercury sphygmomanometer and automated BP monitor. Potential publication bias was determined by Begg's test, and sensitivity analysis was also performed.

\section{Results}

\section{Included studies}

The detailed study selection progress is shown in Fig. 1. Initially, 9642 articles were identified from the PubMed, Web of Science, and Cochrane databases, 26 studies were excluded after browsed the full-text, and 21 original articles were included for the meta-analysis in the end [6, 21-40].

Table 1 shows the characteristics of the 21 articles. Of the 21 articles, all of the studies reported BMI and WHtR, 20 studies reported WC. The total studies were cross-sectional and evaluated 177,943 children and adolescents $3-19$ years of age. The detailed methodological quality assessment of included studies is shown in Fig. 2. 
Table 1

Characteristics of included studies.

\begin{tabular}{|c|c|c|c|c|c|c|c|c|c|}
\hline Study & $\begin{array}{l}\text { Study } \\
\text { year }\end{array}$ & Country & Region & $\begin{array}{l}\text { Study } \\
\text { design }\end{array}$ & Chracteristics & $\begin{array}{l}\text { BP measurement } \\
\text { device }\end{array}$ & $\begin{array}{l}\text { Sample number } \\
\text { (boys/girls) }\end{array}$ & $\begin{array}{l}\text { Age } \\
\text { range } \\
\text { (year) } \\
\text { or } \\
\text { mean } \\
\text { (SD) }\end{array}$ & $\begin{array}{l}\text { Hyperten } \\
\text { criterion } \\
\text { basis }\end{array}$ \\
\hline $\begin{array}{l}\text { Simonetta } \\
\text { [6] }\end{array}$ & 2008 & Italy & Europe & $\begin{array}{l}\text { Cross- } \\
\text { sectional }\end{array}$ & Hypertension & $\begin{array}{l}\text { Mercury } \\
\text { sphygmomanometer }\end{array}$ & 4177(2171/2005) & $5-11$ & $\begin{array}{l}\geq 95 \text { th } \\
\text { percentil } \\
\text { NHBPEP }\end{array}$ \\
\hline Chen [21] & 2011 & China & Asia & $\begin{array}{l}\text { Cross- } \\
\text { sectional }\end{array}$ & Hypertension & $\begin{array}{l}\text { Mercury } \\
\text { sphygmomanometer }\end{array}$ & $939(640 / 299)$ & $3-6$ & $\begin{array}{l}\geq 95 \text { th } \\
\text { percentilı } \\
\text { NHBPEP }\end{array}$ \\
\hline $\begin{array}{l}\text { Arnaud } \\
{[22]}\end{array}$ & 2012 & Switzerland & Europe & $\begin{array}{l}\text { Cross- } \\
\text { sectional }\end{array}$ & $\begin{array}{l}\text { Elevated blood } \\
\text { pressure }\end{array}$ & $\begin{array}{l}\text { Automated BP } \\
\text { monitor }\end{array}$ & $5207(2621 / 2586)$ & $\begin{array}{l}10- \\
15\end{array}$ & $\begin{array}{l}\geq 95 \text { th } \\
\text { percentilı } \\
\text { NHBPEP }\end{array}$ \\
\hline Katrin [23] & 2013 & Germany & Europe & $\begin{array}{l}\text { Cross- } \\
\text { sectional }\end{array}$ & $\begin{array}{l}\text { Elevated blood } \\
\text { pressure }\end{array}$ & $\begin{array}{l}\text { Automated BP } \\
\text { monitor }\end{array}$ & 6813(3492/3321) & $\begin{array}{l}11- \\
17\end{array}$ & $\begin{array}{l}\geq 95 \text { th } \\
\text { percentil } \\
\text { KiGGS }\end{array}$ \\
\hline Anita [24] & 2014 & Italy & Europe & $\begin{array}{l}\text { Cross- } \\
\text { sectional }\end{array}$ & Hypertension & $\begin{array}{l}\text { Mercury } \\
\text { sphygmomanometer }\end{array}$ & $883(455 / 428)$ & $8-18$ & $\begin{array}{l}\geq 95 \text { th } \\
\text { percentilı } \\
\text { NHBPEP }\end{array}$ \\
\hline Dong [25] & 2015 & China & Asia & $\begin{array}{l}\text { Cross- } \\
\text { sectional }\end{array}$ & $\begin{array}{l}\text { Elevated blood } \\
\text { pressure }\end{array}$ & $\begin{array}{l}\text { Mercury } \\
\text { sphygmomanometer }\end{array}$ & $99,366(49514 / 49852)$ & $7-17$ & $\begin{array}{l}\geq 95 \text { th } \\
\text { percentilı } \\
\text { NHBPEP }\end{array}$ \\
\hline Beck [26] & 2011 & Brazil & America & $\begin{array}{l}\text { Cross- } \\
\text { sectional }\end{array}$ & Hypertension & $\begin{array}{l}\text { Mercury } \\
\text { sphygmomanometer }\end{array}$ & $660(343 / 317)$ & $\begin{array}{l}14- \\
19\end{array}$ & $\begin{array}{l}\geq 90 \text { th } \\
\text { percentil } \\
\text { Brazil }\end{array}$ \\
\hline Liang [27] & 2015 & China & Asia & $\begin{array}{l}\text { Cross- } \\
\text { sectional }\end{array}$ & $\begin{array}{l}\text { Elevated blood } \\
\text { pressure }\end{array}$ & $\begin{array}{l}\text { Mercury } \\
\text { sphygmomanometer }\end{array}$ & $5471(2799 / 2672)$ & $6-10$ & $\begin{array}{l}\geq 95 \text { th } \\
\text { percentilı } \\
\text { NHBPEP }\end{array}$ \\
\hline $\begin{array}{l}\text { Mirmiran } \\
\text { [28] }\end{array}$ & 2014 & Iran & Asia & $\begin{array}{l}\text { Cross- } \\
\text { sectional }\end{array}$ & Hypertension & $\begin{array}{l}\text { Mercury } \\
\text { sphygmomanometer }\end{array}$ & $134(66 / 68)$ & $\begin{array}{l}10- \\
18\end{array}$ & $\begin{array}{l}\geq 90 \text { th } \\
\text { percentil } \\
\text { Iran }\end{array}$ \\
\hline $\begin{array}{l}\text { Mishra } \\
{[29]}\end{array}$ & 2015 & India & Asia & $\begin{array}{l}\text { Cross- } \\
\text { sectional }\end{array}$ & Hypertension & $\begin{array}{l}\text { Mercury } \\
\text { sphygmomanometer }\end{array}$ & $1913(1111 / 802)$ & $6-16$ & $\begin{array}{l}\geq 90 \text { th } \\
\text { percentilı } \\
\text { NHBPEP }\end{array}$ \\
\hline Diego [30] & 2017 & Brazil & America & $\begin{array}{l}\text { Cross- } \\
\text { sectional }\end{array}$ & Hypertension & $\begin{array}{l}\text { Automated BP } \\
\text { monitor }\end{array}$ & $8295(4877 / 3418)$ & $\begin{array}{l}10- \\
17\end{array}$ & $\begin{array}{l}\geq 95 \text { th } \\
\text { percentilı } \\
\text { NHBPEP }\end{array}$ \\
\hline $\begin{array}{l}\text { Adeleke } \\
\text { [31] }\end{array}$ & 2018 & Canada & America & $\begin{array}{l}\text { Cross- } \\
\text { sectional }\end{array}$ & Hypertension & $\begin{array}{l}\text { Automated BP } \\
\text { monitor }\end{array}$ & $762(360 / 402)$ & $\begin{array}{l}5.8- \\
17\end{array}$ & $\begin{array}{l}\geq 95 \text { th } \\
\text { percentilı } \\
\text { NHBPEP }\end{array}$ \\
\hline Lu [32] & 2018 & China & Asia & $\begin{array}{l}\text { Cross- } \\
\text { sectional }\end{array}$ & $\begin{array}{l}\text { Elevated blood } \\
\text { pressure }\end{array}$ & $\begin{array}{l}\text { Mercury } \\
\text { sphygmomanometer }\end{array}$ & 1898(955/943) & $7-15$ & $\begin{array}{l}\geq 95 \text { th } \\
\text { percentilı } \\
\text { NHBPEP }\end{array}$ \\
\hline Whye [33] & 2018 & Malaysia & Asia & $\begin{array}{l}\text { Cross- } \\
\text { sectional }\end{array}$ & Hypertension & $\begin{array}{l}\text { Automated BP } \\
\text { monitor }\end{array}$ & $2461(1033 / 1428)$ & $\begin{array}{l}12- \\
17\end{array}$ & $\begin{array}{l}\geq 90 \text { th } \\
\text { percentilı } \\
\text { Brazil }\end{array}$ \\
\hline Joyce [34] & 2019 & Malaysia & Asia & $\begin{array}{l}\text { Cross- } \\
\text { sectional }\end{array}$ & Hypertension & $\begin{array}{l}\text { Automated BP } \\
\text { monitor }\end{array}$ & $513(211 / 302)$ & $\begin{array}{l}12- \\
16\end{array}$ & $\begin{array}{l}\geq 95 \text { th } \\
\text { percentil } \\
\text { Malaysia }\end{array}$ \\
\hline $\begin{array}{l}\text { Renata } \\
{[35]}\end{array}$ & 2019 & Lithuania & Europe & $\begin{array}{l}\text { Cross- } \\
\text { sectional }\end{array}$ & Hypertension & $\begin{array}{l}\text { Automated BP } \\
\text { monitor }\end{array}$ & 7457(3494/3963) & $\begin{array}{l}12- \\
15\end{array}$ & $\begin{array}{l}\geq 95 \text { th } \\
\text { percentil } \\
\text { NHBPEP }\end{array}$ \\
\hline $\begin{array}{l}\text { Chih-Yu } \\
\text { [36] }\end{array}$ & 2020 & China & Asia & $\begin{array}{l}\text { Cross- } \\
\text { sectional }\end{array}$ & Hypertension & $\begin{array}{l}\text { Automated BP } \\
\text { monitor }\end{array}$ & $340(163 / 177)$ & $7-12$ & $\begin{array}{l}\geq 95 \text { th } \\
\text { percentilı } \\
\text { NHBPEP }\end{array}$ \\
\hline Wang [37] & 2019 & China & Asia & $\begin{array}{l}\text { Cross- } \\
\text { sectional }\end{array}$ & Hypertension & $\begin{array}{l}\text { Mercury } \\
\text { sphygmomanometer }\end{array}$ & $683(366 / 317)$ & $8-15$ & $\begin{array}{l}\geq 95 \text { th } \\
\text { percentilı } \\
\text { China }\end{array}$ \\
\hline Li [38] & 2020 & China & Asia & $\begin{array}{l}\text { Cross- } \\
\text { sectional }\end{array}$ & Hypertension & $\begin{array}{l}\text { Mercury } \\
\text { sphygmomanometer }\end{array}$ & $15,698(8004 / 7694)$ & $6-17$ & $\begin{array}{l}\geq 95 \text { th } \\
\text { percentil } \\
\text { China }\end{array}$ \\
\hline
\end{tabular}

BP, blood pressure; BMI, body mass index; WC, waist circumference; WHtR, waist-to-height ratio 


\begin{tabular}{|c|c|c|c|c|c|c|c|c|c|}
\hline Study & $\begin{array}{l}\text { Study } \\
\text { year }\end{array}$ & Country & Region & $\begin{array}{l}\text { Study } \\
\text { design }\end{array}$ & Chracteristics & $\begin{array}{l}\text { BP measurement } \\
\text { device }\end{array}$ & $\begin{array}{l}\text { Sample number } \\
\text { (boys/girls) }\end{array}$ & $\begin{array}{l}\text { Age } \\
\text { range } \\
\text { (year) } \\
\text { or } \\
\text { mean } \\
(\mathrm{SD})\end{array}$ & $\begin{array}{l}\text { Hyperten } \\
\text { criterion } \\
\text { basis }\end{array}$ \\
\hline $\begin{array}{l}\text { Manuel } \\
\text { [39] }\end{array}$ & 2020 & Spain & Europe & $\begin{array}{l}\text { Cross- } \\
\text { sectional }\end{array}$ & Hypertension & $\begin{array}{l}\text { Automated BP } \\
\text { monitor }\end{array}$ & $265(144 / 121)$ & $6-16$ & $\begin{array}{l}\geq 95 \text { th } \\
\text { percentil } \\
\text { AEP }\end{array}$ \\
\hline $\begin{array}{l}\text { Mayram } \\
{[40]}\end{array}$ & 2020 & Iran & Asia & $\begin{array}{l}\text { Cross- } \\
\text { sectional }\end{array}$ & $\begin{array}{l}\text { Hypertension/elevated } \\
\text { blood presure }\end{array}$ & $\begin{array}{l}\text { Mercury } \\
\text { sphygmomanometer }\end{array}$ & 14,008(7091/6917) & $7-18$ & $\begin{array}{l}\geq 90 \text { th; } \\
\geq 95 \text { th } \\
\text { percentil } \\
\text { AAP }\end{array}$ \\
\hline
\end{tabular}

BP, blood pressure; BMI, body mass index; WC, waist circumference; WHtR, waist-to-height ratio

\section{Meta-analysis}

Meta-analysis showed that the pooled area under the reporting receiver-operating characteristic curves (AUC) and 95\% confidence intervals (Cls) for BMI, WC, and WHtR to detect hypertension of boys were $0.68(0.64,0.72), 0.69(0.64,0.74), 0.67(0.63,0.71)$, the pooled AUCs and $95 \%$ Cls of elevated BP were 0.67 $(0.61,0.73), 0.65(0.58,0.73), 0.65(0.61,0.71)$. For girls, the pooled AUCs and $95 \%$ Cls for BMI, WC and WHtR of predicting hypertension were $0.70(0.66,0.75)$, $0.69(0.64,0.75), 0.67(0.63,0.72)$, the pooled AUCs and $95 \%$ Cls of predicting elevated BP were $0.63(0.61,0.65), 0.62(0.60,0.65), 0.62(0.60,0.64)$ respectively. Figure 3 shows the forest plots of AUC scores for hypertension risk (a) and elevated BP risk (b) in boys, heterogeneity was observed across studies ( hypertension : $R=95.7 \%-96.4 \%$; elevated BP: $R=97.6 \%-98.9 \%$ ), Fig. 4 shows the forest plots of AUC scores in girls for hypertension risk (a) and elevated BP risk (b), heterogeneity across studies for hypertension risk was $R=95.7 \%-97.7 \%$; and elevated $\mathrm{BP}$ risk was $R=79.5 \%-86.1 \%$, so randomeffects model was used for BMI, WC, and the WHtR.

For the same anthropometric index, the ability of predicting hypertension and elevated BP was compared in the same gender (Fig. 3 and Fig. 4). The results showed that the AUC value of hypertension in children is higher than elevated BP, the accuracy of BMI in predicting hypertension is significantly higher than elevated BP in girls $(P<0.05)$, while the difference was not obvious in other indices $(P>0.05)$.

\section{Subgroup analysis}

Table 2 shows that the subgroup analysis of pooled AUC values and 95\% Cls of BMI, WC, and the WHtR for hypertension and elevated BP risk. For regional analysis, blood pressure criterion ( $\geq 90$ th percentile and $\geq 95$ th percentile), diagnostic basis (NHBPEP and other local criteria of hypertension) and BP measurement device, there was no statistical significance existed between BMI, WC, and the WHtR for hypertension and elevated BP risk. 
Table 2

Subgroup analysis of pooled AUC values with $95 \% \mathrm{Cl}$ in pediatric population.

\begin{tabular}{|c|c|c|c|c|c|c|c|c|c|}
\hline \multirow{3}{*}{$\begin{array}{l}\text { Subgroup } \\
\text { (N of studies) }\end{array}$} & \multicolumn{4}{|l|}{ Hypertension } & \multirow{3}{*}{$\begin{array}{l}\text { Subgroup } \\
\text { ( } \mathrm{N} \text { of studies) }\end{array}$} & \multicolumn{4}{|c|}{ Elevated blood pressure } \\
\hline & \multicolumn{2}{|l|}{ Boys } & \multicolumn{2}{|l|}{ Girls } & & \multicolumn{2}{|l|}{ Boys } & \multicolumn{2}{|l|}{ Girls } \\
\hline & $\mathrm{AUC}(95 \% \mathrm{Cl})$ & $R(\%)$ & $\mathrm{AUC}(95 \% \mathrm{Cl})$ & $R(\%)$ & & $\mathrm{AUC}(95 \% \mathrm{Cl})$ & $R(\%)$ & $\mathrm{AUC}(95 \% \mathrm{Cl})$ & $R(\%)$ \\
\hline \multicolumn{5}{|l|}{ Region } & \multicolumn{5}{|l|}{ Region } \\
\hline \multicolumn{5}{|l|}{ Asia } & \multicolumn{5}{|l|}{ Asia } \\
\hline BMI $(n=9)$ & $0.67(0.63,0.72)$ & 93.1 & $0.68(0.63,0.74)$ & 92.5 & $\mathrm{BMI}(\mathrm{n}=4)$ & $0.68(0.60,0.76)$ & 98.8 & $0.64(0.62,0.67)$ & 84.7 \\
\hline$W C(n=8)$ & $0.67(0.61,0.73)$ & 94.1 & $0.68(0.62,0.74)$ & 94.3 & $W C(n=4)$ & $0.66(0.58,0.76)$ & 99.2 & $0.63(0.60,0.66)$ & 88.9 \\
\hline $\mathrm{WHtR}(\mathrm{n}=9)$ & $0.67(0.62,0.71)$ & 91.4 & $0.66(0.61,0.72)$ & 93.6 & $\mathrm{WHtR}(\mathrm{n}=4)$ & $0.66(0.60,0.73)$ & 98.5 & $0.62(0.59,0.65)$ & 89.0 \\
\hline \multicolumn{5}{|l|}{ Europe } & \multicolumn{5}{|l|}{ Europe } \\
\hline BMI $(n=4)$ & $0.73(0.64,0.82)$ & 93.1 & $0.70(0.63,0.77)$ & 90.9 & $\mathrm{BMI}(\mathrm{n}=2)$ & $0.65(0.59,0.72)$ & 91.0 & $0.62(0.60,0.64)$ & 0.0 \\
\hline$W C(n=4)$ & $0.72(0.64,0.81)$ & 91.9 & $0.68(0.62,0.74)$ & 83.4 & WC $(n=1)$ & $0.61(0.58,0.64)$ & - & $0.61(0.58,0.64)$ & - \\
\hline $\mathrm{WHtR}(\mathrm{n}=4)$ & $0.66(0.55,0.79)$ & 95.5 & $0.65(0.58,0.73)$ & 89.8 & WHtR $(n=2)$ & $0.64(0.60,0.69)$ & 83.0 & $0.61(0.59,0.64)$ & 0.0 \\
\hline \multicolumn{5}{|l|}{ America } & \multicolumn{5}{|l|}{ America } \\
\hline $\mathrm{BMI}(\mathrm{n}=3)$ & $0.66(0.57,0.76)$ & 95.1 & $0.77(0.55,1.00)$ & 98.9 & - & - & - & - & - \\
\hline WC $(n=3)$ & $0.71(0.57,0.90)$ & 96.9 & $0.75(0.52,1.00)$ & 99.4 & - & - & - & - & - \\
\hline $\mathrm{WHtR}(\mathrm{n}=3)$ & $0.69(0.54,0.88)$ & 96.4 & $0.73(0.52,1.00)$ & 98.7 & - & - & - & - & - \\
\hline \multicolumn{5}{|c|}{ Hypertension criterion } & \multicolumn{5}{|c|}{ Elevated blood pressure criterion } \\
\hline \multicolumn{5}{|c|}{$\geq 90$ th percentile } & \multicolumn{5}{|c|}{$\geq 90$ th percentile } \\
\hline BMI $(n=4)$ & $0.68(0.63,0.74)$ & 90.8 & $0.77(0.66,0.91)$ & 93.6 & $\mathrm{BMI}(\mathrm{n}=1)$ & $0.56(0.54,0.59)$ & - & $0.59(0.56,0.62)$ & - \\
\hline$W C(n=4)$ & $0.72(0.64,0.82)$ & 89.9 & $0.75(0.61,0.93)$ & 91.3 & WC $(n=1)$ & $0.54(0.52,0.57)$ & - & $0.57(0.54,0.60)$ & - \\
\hline $\mathrm{WHtR}(\mathrm{n}=4)$ & $0.72(0.64,0.80)$ & 84.4 & $0.75(0.64,0.87)$ & 93.6 & WHtR $(n=1)$ & $0.56(0.54,0.59)$ & - & $0.56(0.53,0.59)$ & - \\
\hline \multicolumn{5}{|c|}{$\geq 95$ th percentile } & \multicolumn{5}{|c|}{$\geq 95$ th percentile } \\
\hline BMI $(n=12)$ & $0.68(0.64,0.74)$ & 96.9 & $0.68(0.64,0.72)$ & 94.5 & $\mathrm{BMI}(\mathrm{n}=5)$ & $0.69(0.63,0.75)$ & 97.9 & $0.64(0.62,0.66)$ & 65.1 \\
\hline$W C(n=11)$ & $0.68(0.63,0.73)$ & 96.7 & $0.67(0.63,0.71)$ & 93.7 & $W C(n=4)$ & $0.68(0.60,0.77)$ & 99.0 & $0.64(0.62,0.66)$ & 74.8 \\
\hline WHtR $(n=12)$ & $0.65(0.61,0.70)$ & 94.9 & $0.64(0.61,0.68)$ & 92.9 & WHtR $(n=5)$ & $0.68(0.63,0.73)$ & 97.3 & $0.63(0.62,0.65)$ & 49.1 \\
\hline \multicolumn{5}{|c|}{ Hypertension diagnosis basis } & \multicolumn{5}{|c|}{ Elevated blood pressure diagnosis basis } \\
\hline NHBPEP & & & & & NHBPEP & & & & \\
\hline BMI $(n=8)$ & $0.69(0.63,0.75)$ & 97.6 & $0.67(0.62,0.73)$ & 94.1 & $\mathrm{BMI}(\mathrm{n}=4)$ & $0.69(0.62,0.77)$ & 98.5 & $0.65(0.63,0.67)$ & 68.7 \\
\hline WC $(n=7)$ & $0.67(0.60,0.74)$ & 97.5 & $0.65(0.61,0.70)$ & 91.3 & WC $(n=4)$ & $0.68(0.60,0.77)$ & 99.0 & $0.64(0.62,0.66)$ & 74.8 \\
\hline WHtR $(n=8)$ & $0.64(0.59,0.70)$ & 94.7 & $0.64(0.59,0.68)$ & 91.9 & WHtR $(n=4)$ & $0.68(0.62,0.74)$ & 98.0 & $0.64(0.63,0.65)$ & 32.0 \\
\hline Other local crite & ria of hypertension & & & & Other local crit & ria of elevated blo & d press & & \\
\hline $\mathrm{BMI}(\mathrm{n}=8)$ & $0.68(0.62,0.74)$ & 94.0 & $0.73(0.65,0.82)$ & 96.5 & $\mathrm{BMI}(\mathrm{n}=2)$ & $0.62(0.51,0.75)$ & 97.5 & $0.60(0.57,0.62)$ & 0.0 \\
\hline WC $(n=8)$ & $0.71(0.65,0.77)$ & 93.8 & $0.73(0.63,0.84)$ & 98.2 & $W C(n=1)$ & $0.54(0.52,0.57)$ & - & $0.57(0.54,0.60)$ & - \\
\hline $\mathrm{WHtR}(\mathrm{n}=8)$ & $0.70(0.64,0.76)$ & 92.3 & $0.71(0.63,0.80)$ & 96.6 & WHtR $(n=2)$ & $0.61(0.51,0.73)$ & 96.7 & $0.58(0.54,0.63)$ & 73.7 \\
\hline BP measureme & nt device & & & & BP measurem & tt device & & & \\
\hline Mercury sphyg & nomanometer & & & & Mercury sphyg & nomanometer & & & \\
\hline $\mathrm{BMI}(\mathrm{n}=9)$ & $0.66(0.62,0.71)$ & 93.6 & $0.70(0.63,0.77)$ & 95.2 & $\mathrm{BMI}(\mathrm{n}=4)$ & $0.68(0.60,0.76)$ & 98.8 & $0.64(0.62,0.67)$ & 84.7 \\
\hline$W C(n=9)$ & $0.67(0.61,0.73)$ & 93.8 & $0.68(0.60,0.78)$ & 97.9 & WC $(n=4)$ & $0.66(0.58,0.76)$ & 99.2 & $0.63(0.60,0.66)$ & 88.9 \\
\hline $\mathrm{WHtR}(\mathrm{n}=9)$ & $0.66(0.62,0.71)$ & 90.3 & $0.66(0.60,0.73)$ & 94.5 & WHtR $(n=4)$ & $0.66(0.60,0.73)$ & 98.5 & $0.62(0.59,0.65)$ & 89.0 \\
\hline Automated BP & monitor & & & & Automated BP & honitor & & & \\
\hline $\mathrm{BMI}(\mathrm{n}=7)$ & $0.72(0.64,0.80)$ & 97.9 & $0.71(0.64,0.79)$ & 96.8 & $\mathrm{BMI}(\mathrm{n}=2)$ & $0.65(0.59,0.72)$ & 91.0 & $0.62(0.60,0.64)$ & 0.0 \\
\hline
\end{tabular}

AUC, area under the curve; $\mathrm{Cl}$, confidence interval; BMI, body mass index; WC, waist circumference; WHtR, waist-to height ratio. 


\begin{tabular}{|c|c|c|c|c|c|c|c|c|c|}
\hline \multirow{3}{*}{$\begin{array}{l}\text { Subgroup } \\
\text { ( } \mathrm{N} \text { of studies) }\end{array}$} & \multicolumn{4}{|l|}{ Hypertension } & \multirow{3}{*}{$\begin{array}{l}\text { Subgroup } \\
\text { ( } N \text { of studies) }\end{array}$} & \multicolumn{4}{|c|}{ Elevated blood pressure } \\
\hline & \multicolumn{2}{|l|}{ Boys } & \multicolumn{2}{|l|}{ Girls } & & \multicolumn{2}{|l|}{ Boys } & \multicolumn{2}{|l|}{ Girls } \\
\hline & $\mathrm{AUC}(95 \% \mathrm{Cl})$ & $R(\%)$ & $\mathrm{AUC}(95 \% \mathrm{Cl})$ & $R(\%)$ & & $\operatorname{AUC}(95 \% \mathrm{Cl})$ & $R(\%)$ & AUC( $95 \% \mathrm{Cl})$ & $R(\%)$ \\
\hline$W C(n=6)$ & $0.72(0.64,0.81)$ & 98.1 & $0.71(0.63,0.80)$ & 97.2 & WC $(n=1)$ & $0.61(0.58,0.64)$ & - & $0.61(0.58,0.64)$ & - \\
\hline WHtR $(n=7)$ & $0.68(0.60,0.76)$ & 97.3 & $0.68(0.61,0.77)$ & 96.9 & WHtR $(n=2)$ & $0.64(0.60,0.69)$ & 83.0 & $0.61(0.59,0.64)$ & 0.0 \\
\hline
\end{tabular}

\section{Publication bias and sensitivity analysis}

Table 3 shows that no publication bias in hypertension and elevated BP were found according to the Begg's test (hypertension-boys: $P=0.964$, girls: $P=0.444$ for BMl; boys: $P=0.692$, girls: $P=0.198$ for WC; boys: $P=0.558$, girls: $P=0.260$ for the WHtR; elevated BP-boys: $P=1.000$, girls: $P=0.452$ for BMl; boys: $P=$ 0.462 , girls: $P=0.806$ for WC; boys: $P=1.000$, girls: $P=0.260$ for the WHtR). Sensitivity analysis was carried out and each study was excluded separately. The outcomes were not altered significantly when excluded. Indeed, none of the studies had a significant impact on the overall results.

Table 3

Results of publication bias.

\begin{tabular}{|llll|}
\hline $\begin{array}{l}\text { Anthropometric } \\
\text { indices }\end{array}$ & $\mathrm{N}$ of studies & Gender & Begg's $\boldsymbol{P}$ \\
\hline BMI & 16 & boys & 0.964 \\
\hline WC & 16 & girls & 0.444 \\
\hline WHtR & 15 & boys & 0.692 \\
\hline BMI & 15 & girls & 0.198 \\
\hline & 16 & boys & 0.558 \\
\hline WC & 16 & girls & 0.260 \\
\hline & 6 & boys & 1.000 \\
\hline WHtR & 6 & girls & 0.452 \\
\hline & 5 & boys & 0.462 \\
\hline
\end{tabular}

\section{Discussion}

This robust meta-analysis, which included 177,943 children and adolescents 3-19 years of age from populations all over the world, our studies comparison in the pediatric population showed that there is no statistical difference in the accuracy of BMI, WC, WHtR in screening children or adolescents for hypertension and elevated blood pressure. The Chunming meta-analysis [12] of 9 cross-sectional studies included 25,424 children and adolescents $6-18$ years of age in 7 different countries and confirmed that no anthropometric index was statistically superior to other anthropometric indices in identifying an elevated BP; on the basis of pooled AUC values, the AUC values were ranked in the following order, but this was not statistically significant: $\mathrm{BMI}(0.778)>\mathrm{WC}(0.718)>\mathrm{WHtR}$ (0.670). With the increased number of included articles in the current systematic review and increased number of subjects $(n=177,943)$, apparently, we obtained the same conclusion.

According to the Chunming meta-analysis [12], the number of included studies was less, and which only included the studies based on NHBPEP as the diagnosis basis of hypertension. However, in terms of anthropometric index prediction, our study includes two characteristics of hypertension and elevated blood pressure, which means that we have analyzed both for the results of hypertension and the process of elevated blood pressure. Besides, the study not only included the studies based on NHBPEP as the diagnosis basis of hypertension, but also contained other local criteria of hypertension, because there is no clear and unified standard for the diagnosis of hypertension in children, some local hypertension diagnostic criteria are also applicable to local children and adolescents, which greatly improved the comprehensiveness of the included studies. In terms of subgroup analysis, we comprehensively consider the factors that may have an impact on results, and conducted subgroup analysis including region, blood pressure criterion, diagnostic basis and blood pressure measurement device.

Both adults and children with overweight or obesity are associated with elevated BP [12,41,42] and weight loss can also improve BP levels in children with obesity $[43,44]$. BMI, WC, and the WHtR are common indicators for predicting hypertension in children, the current meta-analysis suggested that the above three anthropometrics are all positively related to pediatric elevated blood pressure (AUC >0.5). Among the anthropometric indices, BMI is most commonly used $[45,46]$ owing to its simplicity. However, the BMI also has shortcomings because BMI cannot distinguish between individuals with fattiness and individuals with muscle mass and cannot determine the location of fattiness [47, 48]. WC is often used to reflect abdominal obesity due to its strongly 
relationship with visceral fat depots [45]. It has been reported that body fat distribution has a close relationship to the occurrence and development of cardiovascular disease [49]. Visceral adipose tissue (VAT) accumulation, which is associated with an increase in free fatty acid content and insulin resistance, increases the risk of hypertension $[50,51]$. WC and the WHtR are strongly related to abdominal obesity and VAT, which were assessed by radiologic examination [52,53]. Therefore, a meta-analysis of adults showed that the WHtR is superior to WC and BMI in screening cardiovascular diseases [54], which confirmed that the index reflecting abdominal obesity is better than BMI in predicting metabolic risk. However, contrary to the research findings in adults, the analytical results in our review showed that no significant difference in BMI, WC and WHtR was found in predicting the risk of pediatric hypertension. This finding may reflect the rapid growth in stature from childhood to adolescence that outweighs the incremental change in WC, which greatly reduces the superiority of WHtR over WC and BMI to predict abdominal obesity [55]. However, our review showed that the ability of all the indicators to predict hypertension is higher than elevated blood pressure, especially BMI in girls. Limited by the number of included studies may lead to this result, another possible reason may be the early small increase in blood pressure in children is more secretive and difficult to find. Only when the blood pressure increases significantly and exceeds the critical value, can be easier to predict through anthropometric indicators. A previous study suggested that the WHtR decreases with age from $5-$ 16 years until 18 years due to the cessation in growth [56]. That finding may also misclassify fast-growing children with excess abdominal fat as healthy. In addition, some studies involving children and adolescents showed that, unlike adults, BMI offers adequate information to evaluate visceral obesity and WC does not predict extra information [57]. Another study reported that the 95th BMI percentile of the Center for Disease Control is a useful threshold for predicting increased VAT, fattiness, and heart metabolic risk in children and adolescents [58].

ROC analysis has been a widely-used method for evaluating the efficacy of diagnostic tests. This meta-analysis focused on studies that used ROC method to compare the discriminatory power of anthropometric indices for elevated BP. However, there are still some limitations in this systematic review. First, the most important limitation is the different criteria of hypertension in the included studies. Most studies used NHBPEP criterion, and some studies applied local criterion. Therefore, both diagnostic standards were included and the subgroup analysis was conducted to explore the impact of anthropometric indicators predicting high BP and elevated BP. Some of them used the 90th percentile as a cutoff to diagnose hypertension and elevated BP, and some used the 95th percentile as a cutoff, so all the cutoff were included for subgroup analysis to explore further differences. Second, our study only considered three obesity anthropometric indices, yet other indices, such as the neck circumference [58], waist-to-hip ratio, and mid-upper arm circumference [59], were not used in this study because of the limited number of relevant papers. Finally, most results have the problem of high heterogeneity, though we have conducted the subgroup analysis to explore the possible sources of heterogeneity, the degree of heterogeneity did not decrease.

\section{Conclusion}

In conclusion, this systematic review with a meta-analysis showed that no anthropometric index was statistically superior to other anthropometric indices in identifying hypertension and elevated BP in adolescents. However, for the same anthropometric index, the accuracy in predicting hypertension is better than elevated blood pressure, especially the application of BMI in girls. Further exploration is needed to find better anthropometric indicators for predicting early blood pressure abnormalities in children.

\section{Abbreviations}

BP: Blood pressure; ROC: Receiver operating characteristics; BMI: Body mass index; WC: Waist circumference; WHtR: Waist-to-height ratio; AUC: Area under curve; SBP: Systolic blood pressure; DBP: Diastolic blood pressure. NHBPEP: National high blood pressure education program; QUADAS-2: Quality Assessment of Diagnostic Accuracy Studies-2; VAT: Visceral adipose tissue.

\section{Declarations}

Ethics approval and consent to participate: Not applicable.

Consent for publication: Not applicable.

Availability of data and materials: The datasets generated and analysed during the current study are available from the corresponding author on reasonable request.

Competing interests: The authors declare that they have no competing interests.

Funding: Not applicable.

Authors' contributions: Jun-Min Tao conceived data, wrote the original draft and performed the primary analysis. Wei Wei and Xiao-Yang Ma extracted the data and helped with the analysis. Ying-Xiang Huo and Meng-Die Hu performed the formal analysis. Xiao-Feng Li and Xin Chen edited the review \& editing and provided methodology. All authors reviewed and revised the manuscript. Xin Chen acts as guarantor. The authors read and approved the final manuscript.

Acknowledgement: Not applicable.

\section{References}

1. Whitworth JA. 2003 World Health Organization (WHO)/International Society of Hypertension (ISH) statement on management of hypertension. J Hypertens. 2003;21:1983-92. https://doi.org/10.1097/00004872-200311000-00002 
2. Dobson CP, Eide M, Nylund CM. Hypertension Prevalence, Cardiac Complications, and Antihypertensive Medication Use in Children. J Pediatr. 2015;167:92 - 7.e1. https://doi.org/10.1016/j.jpeds.2015.04.016

3. Litwin M, Feber J, Ruzicka M. Vascular Aging: Lessons From Pediatric Hypertension. Can J Cardiol. 2016;32:642-9. https://doi.org/10.1016/j.cjca.2016.02.064

4. Chen L, Simonsen N, Liu L. Racial Differences of Pediatric Hypertension in Relation to Birth Weight and Body Size in the United States. PloS One. 2015;10:e0132606. https://doi.org/10.1371/journal.pone.0132606

5. Kelly RK, Thomson R, Smith KJ, Dwyer T, Venn A, Magnussen CG. Factors Affecting Tracking of Blood Pressure from Childhood to Adulthood: The Childhood Determinants of Adult Health Study. J Pediatr. 2015;167:1422-8

.e2 . https://doi.org/10.1016/j.jpeds.2015.07.055

6. Genovesi S, Antolini L, Giussani M, Pieruzzi F, Galbiati S, Valsecchi MG, et al. Usefulness of waist circumference for the identification of childhood hypertension. J Hypertens. 2008;26:1563-70. https://doi.org/10.1097/HJH.0b013e328302842b

7. Sorof J, Daniels S. Obesity hypertension in children: a problem of epidemic proportions. Hypertension. 2002;40:441-7. https://doi.org/10.1161/01.hyp.0000032940.33466.12

8. Rosner B, Prineas R, Daniels SR, Loggie J. Blood pressure differences between blacks and whites in relation to body size among US children and adolescents. Am J Epidemiol. 2000;151:1007-19. https://doi.org/10.1093/oxfordjournals.aje.a010129

9. de Onis M, Blössner M, Borghi E. Global prevalence and trends of overweight and obesity among preschool children. Am J Clin Nutr. 2010;92:1257-64. https://doi.org/10.1093/oxfordjournals.aje.a010129

10. Rokholm B, Baker JL, Sørensen TI. The levelling off of the obesity epidemic since the year 1999-a review of evidence and perspectives. Obes Rev. 2010;11:835-46. https://doi.org/10.1111/j.1467-789X.2010.00810.x

11. Benjamin RM. The Surgeon General's vision for a healthy and fit nation. Public Health Reps. 2010;125:514-5. https://doi.org/10.1177/003335491012500402

12. Ma C, Wang R, Liu Y, Lu Q, Lu N, Tian Y, et al. Performance of obesity indices for screening elevated blood pressure in pediatric population: Systematic review and meta-analysis. Medicine. 2016;95:e4811. https://doi.org/10.1097/md.0000000000004811

13. Moher D, Liberati A, Tetzlaff J, Altman DG. Preferred reporting items for systematic reviews and meta-analyses: the PRISMA statement. PLoS Med. 2009;6:e1000097. https://doi.org/10.1371/journal.pmed.1000097

14. Physical status: the use and interpretation of anthropometry. Report of a WHO Expert Committee. World Health Organization technical report series. 1995;854:1-452.

15. The fourth report on the diagnosis, evaluation, and treatment of high blood pressure in children and adolescents. Pediatrics. 2004;114:555-76.

16. Whiting PF, Rutjes AW, Westwood ME, Mallett S, Deeks JJ, Reitsma JB, et al. QUADAS-2: a revised tool for the quality assessment of diagnostic accuracy studies. Ann Intern Med. 2011;155:529-36. https://doi.org/10.1371/journal.pmed.1000097

17. Browning LM, Hsieh SD, Ashwell M. A systematic review of waist-to-height ratio as a screening tool for the prediction of cardiovascular disease and diabetes: 0.5 could be a suitable global boundary value. Nutr Res Rev. 2010;23:247-69. https://doi.org/10.1371/journal.pmed.1000097

18. Fokom-Domgue J, Combescure C, Fokom-Defo V, Tebeu PM, Vassilakos P, Kengne AP, et al. Performance of alternative strategies for primary cervical cancer screening in sub-Saharan Africa: systematic review and meta-analysis of diagnostic test accuracy studies. BMJ. 2015;351:h3084. https://doi.org/10.1371/journal.pmed.1000097

19. Barry E, Galvin R, Keogh C, Horgan F, Fahey T. Is the Timed Up and Go test a useful predictor of risk of falls in community dwelling older adults: a systematic review and meta-analysis. BMC Geriatr. 2014;14:14. https://doi.org/10.1186/1471-2318-14-14

20. Rubin KH, Friis-Holmberg T, Hermann AP, Abrahamsen B, Brixen K. Risk assessment tools to identify women with increased risk of osteoporotic fracture: complexity or simplicity? A systematic review. J Bone Miner Res. 2013;28:1701-17. https://doi.org/10.1002/jbmr.1956

21. Chen B, Li HF. Waist circumference as an indicator of high blood pressure in preschool obese children. Asia Pac J Clin Nutr. 2011;20:557-62.

22. Chiolero A, Paradis G, Maximova K, Burnier M, Bovet P. No use for waist-for-height ratio in addition to body mass index to identify children with elevated blood pressure. Blood Press. 2013;22:17-20. https://doi.org/10.3109/08037051.2012.701376

23. Kromeyer-Hauschild K, Neuhauser H, Schaffrath Rosario A, Schienkiewitz A. Abdominal obesity in German adolescents defined by waist-to-height ratio and its association to elevated blood pressure: the KiGGS study. Obes Facts. 2013;6:165-75. https://doi.org/10.3109/08037051.2012.701376

24. Morandi A, Miraglia Del Giudice E, Martino F, Martino E, Bozzola M, Maffeis C. Anthropometric indices are not satisfactory predictors of metabolic comorbidities in obese children and adolescents. J Pediatr. 2014;165:1178-83

.e2 . https://doi.org/10.3109/08037051.2012.701376

25. Dong B, Wang Z, Wang HJ, Ma J. Associations between adiposity indicators and elevated blood pressure among Chinese children and adolescents. J Hum Hypertens. 2015;29:236-40. https://doi.org/10.1038/jhh.2014.95

26. Beck CC, Lopes Ada S, Pitanga FJ. Anthropometric indicators as predictors of high blood pressure in adolescents. Arq Bras Cardiol. 2011;96:126-33. https://10.1590/s0066-782x2010005000153

27. Liang JJ, Chen YJ, Jin Y, Yang WH, Mai JC, Ma J, et al. Comparison of adiposity measures in the identification of children with elevated blood pressure in Guangzhou, China. J Hum Hypertens. 2015;29:732-6. https://doi.org/10.1038/jhh.2015.16 
28. Mirmiran P, Rezaei M, Asghari G, Mehrabi Y, Azizi F. Association of Metabolic Syndrome with Body Fat Percent, Anthropometric Indices in 10 To 18 Year Old Adolescents. Iran J Public Health. 2014;43:193-201.

29. Mishra PE, Shastri L, Thomas T, Duggan C, Bosch R, McDonald CM, et al. Waist-to-Height Ratio as an Indicator of High Blood Pressure in Urban Indian School Children. Indian Pediatr. 2015;52:773-8. https://10.1007/s13312-015-0715-x

30. Christofaro DGD, Farah BQ, Vanderlei LCM, Delfino LD, Tebar WR, Barros MVG, et al. Analysis of different anthropometric indicators in the detection of high blood pressure in school adolescents: a cross-sectional study with 8295 adolescents. Braz J Phys Ther. 2018;22:49-54.

https://doi.org/10.1016/j.bjpt.2017.10.007

31. Fowokan AO, Punthakee Z, Waddell C, Rosin M, Morrison KM, Gupta M, et al. Adiposity measures and their validity in estimating risk of hypertension in South Asian children: a cross-sectional study. BMJ Open. 2019;9:e024087. https://doi.org/10.1136/bmjopen-2018-024087

32. Lu Y, Luo B, Xie J, Zhang X, Zhu H. Prevalence of hypertension and prehypertension and its association with anthropometrics among children: a crosssectional survey in Tianjin, China. J Hum Hypertens. 2018;32:789-98. https://doi.org/10.1038/s41371-018-0088-4

33. Cheah WL, Chang CT, Hazmi H, Kho GWF. Using Anthropometric Indicator to Identify Hypertension in Adolescents: A Study in Sarawak, Malaysia. Int J Hypertens. 2018;2018:6736251. https://doi.org/10.1155/2018/6736251

34. Tee JYH, Gan WY, Lim PY. Comparisons of body mass index, waist circumference, waist-to-height ratio and a body shape index (ABSI) in predicting high blood pressure among Malaysian adolescents: a cross-sectional study. BMJ Open. 2020;10:e032874. https://doi.org/10.1136/bmjopen-2019-032874

35. Renata Kuciene VD. Associations between body mass index, waist circumference, waist-to-height ratio, and high blood pressure among adolescents: a cross-sectional study. Sci Rep. 2019;9:9493. https://doi.org/10.1038/s41598-019-45956-9

36. Hsu CY, Lin RH, Lin YC, Chen JY, Li WC, Lee LA, et al. Are Body Composition Parameters Better than Conventional Anthropometric Measures in Predicting Pediatric Hypertension? Int J Environ Res Public Health. 2020;17. https://doi.org/10.3390/ijerph17165771

37. Wang Y, Liu W, Sun L, Zhang Y, Wang B, Yuan Y, et al. A novel indicator, childhood lipid accumulation product, is associated with hypertension in Chinese children and adolescents. Hypertens Res. 2020;43:305-12. https://10.1038/s41440-019-0366-8

38. Li Y, Zou Z, Luo J, Ma J, Ma Y, Jing J, et al. The predictive value of anthropometric indices for cardiometabolic risk factors in Chinese children and adolescents: A national multicenter school-based study. PloS One. 2020;15:e0227954. https://doi.org/10.1371/journal.pone.0227954

39. Vaquero-Álvarez M, Molina-Luque R, Fonseca-Pozo FJ, Molina-Recio G, López-Miranda J, Romero-Saldaña M. Diagnostic Precision of Anthropometric Variables for the Detection of Hypertension in Children and Adolescents. Int J Environ Res Public Health. 2020;17.

https://doi.org/10.3390/ijerph17124415

40. Yazdi M, Assadi F, Qorbani M, Daniali SS, Heshmat R, Esmaeil Motlagh M, et al. Validity of anthropometric indices in predicting high blood pressure risk factors in Iranian children and adolescents: CASPIAN-V study. J Clin Hypertens. 2020;22:1009-17. https://doi.org/10.1111/jch.13895

41. Wang J, Zhu Y, Jing J, Chen Y, Mai J, Wong SH, et al. Relationship of BMI to the incidence of hypertension: a 4 years' cohort study among children in Guangzhou, 2007-2011. BMC Public Health. 2015;15:782. https://doi.org/10.1186/s12889-015-1997-6

42. Pazin DC, Rosaneli CF, Olandoski M, Oliveira ERN, Baena CP, Figueredo AS, et al. Waist Circumference is Associated with Blood Pressure in Children with Normal Body Mass Index: A Cross-Sectional Analysis of 3,417 School Children. Arq Bras Cardiol. 2017;109:509-15.

https://doi.org/10.5935/abc.20170162

43. Kalarchian MA, Levine MD, Arslanian SA, Ewing LJ, Houck PR, Cheng Y, et al. Family-based treatment of severe pediatric obesity: randomized, controlled trial. Pediatrics. 2009;124:1060-8. https://doi.org/10.1542/peds.2008-3727

44. Shalitin S, Ashkenazi-Hoffnung L, Yackobovitch-Gavan M, Nagelberg N, Karni Y, Hershkovitz E, et al. Effects of a twelve-week randomized intervention of exercise and/or diet on weight loss and weight maintenance, and other metabolic parameters in obese preadolescent children. Horm Res. 2009;72:287301. https://doi.org/10.1159/000245931

45. Chung W, Park CG, Ryu OH. Association of a New Measure of Obesity with Hypertension and Health-Related Quality of Life. PloS One. 2016;11:e0155399. https://doi.org/10.1371/journal.pone.0155399

46. Oreopoulos A, Padwal R, McAlister FA, Ezekowitz J, Sharma AM, Kalantar-Zadeh K, et al. Association between obesity and health-related quality of life in patients with coronary artery disease. Int J Obes (2005). 2010;34:1434-41. https://doi.org/10.1038/ijo.2010.73

47. Son YJ, Kim J, Park HJ, Park SE, Park CY, Lee WY, et al. Association of Waist-Height Ratio with Diabetes Risk: A 4-Year Longitudinal Retrospective Study. Endocrinol Metab (Seoul, Korea). 2016;31:127-33. https://doi.org/10.3803/EnM.2016.31.1.127

48. Heymsfield SB, Scherzer R, Pietrobelli A, Lewis CE, Grunfeld C. Body mass index as a phenotypic expression of adiposity: quantitative contribution of muscularity in a population-based sample. Int J Obes (2005). 2009;33:1363-73. https://doi.org/10.1038/ijo.2009.184

49. Després JP. Body fat distribution and risk of cardiovascular disease: an update. Circulation. 2012;126:1301-13. https://doi.org/10.1038/ijo.2009.184

50. Adler-Wailes DC, Periwal V, Ali AH, Brady SM, McDuffie JR, Uwaifo GI, et al. Sex-associated differences in free fatty acid flux of obese adolesc. J Clin Endocrinol Metab. 2013;98:1676-84. https://doi.org/10.1210/jc.2012-3817

51. Kim JA, Park HS. Association of abdominal fat distribution and cardiometabolic risk factors among obese Korean adolescents. Diabetes Metab. 2008;34:126-30. https://doi.org/10.1016/j.diabet.2007.10.012

52. Savgan-Gurol E, Bredella M, Russell M, Mendes N, Klibanski A, Misra M. Waist to hip ratio and trunk to extremity fat (DXA) are better surrogates for IMCL and for visceral fat respectively than for subcutaneous fat in adolescent girls. Nutr Metab. 2010;7:86. https://doi.org/10.1186/1743-7075-7-86

53. O'Connor M, Ryan J, Foley S. Best single-slice location to measure visceral adipose tissue on paediatric CT scans and the relationship between anthropometric measurements, gender and VAT volume in children. Br J Radiol. 2015;88:20140711. https://doi.org/10.1259/bjr.20140711 
54. Ashwell M, Gunn P, Gibson S. Waist-to-height ratio is a better screening tool than waist circumference and BMI for adult cardiometabolic risk factors: systematic review and meta-analysis. Obes Rev. 2012;13:275-86. https://doi.org/10.1111/j.1467-789X.2011.00952.x

55. Kryst $Ł$, Woronkowicz A, Kowal M, Pilecki MW, Sobiecki J. Abdominal obesity screening tools in the aspects of secular trend. Anthropol Anz. 2016;73:295312. https://doi.org/10.1127/anthranz/2016/0622

56. McCarthy HD, Ashwell M. A study of central fatness using waist-to-height ratios in UK children and adolescents over two decades supports the simple message-'keep your waist circumference to less than half your height'. Int J Obes (2005). 2006;30:988 - 92. https://doi.org/10.1038/sj.ijo.0803226

57. Koren D, Marcus CL, Kim C, Gallagher PR, Schwab R, Bradford RM, et al. Anthropometric predictors of visceral adiposity in normal-weight and obese adolescents. Pediatr Diabetes. 2013;14:575-84. https://doi.org/10.1111/pedi.12042

58. Kuciene R, Dulskiene V, Medzioniene J. Association of neck circumference and high blood pressure in children and adolescents: a case-control study. BMC Pediatr. 2015;15:127. https://doi.org/10.1186/s12887-015-0444-2

59. Ma CM, Li Y, Gao GQ, Yin FZ, Wang R, Liu XL, et al. Mid-upper arm circumference as a screening measure for identifying children with hypertension. Blood Press Monit. 2015;20:189-93. https://10.1097/mbp.0000000000000122

\section{Figures}

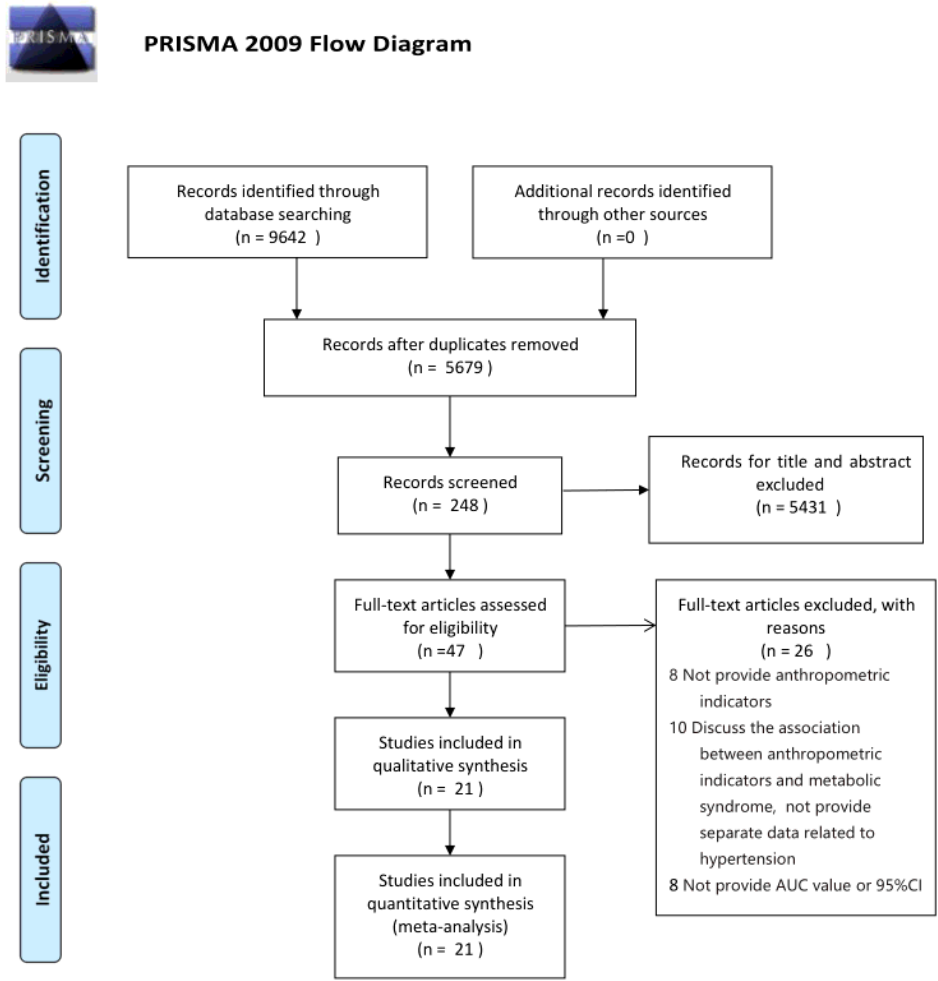

From: Moher D, Liberati A, Tetzlaff J, Aitman DG, The PRISMA Group (2009), Preferred Reporting feems for Systematic Reviews and Meta-
Analyses: The PRISMA Statement. PLoS Med 6(7): 1000097, doi:10.1371/joumal.pmedio00097

For more information, visit www. prisma-statement.org.

Figure 1

Prisma 2009 Flow Diagram 


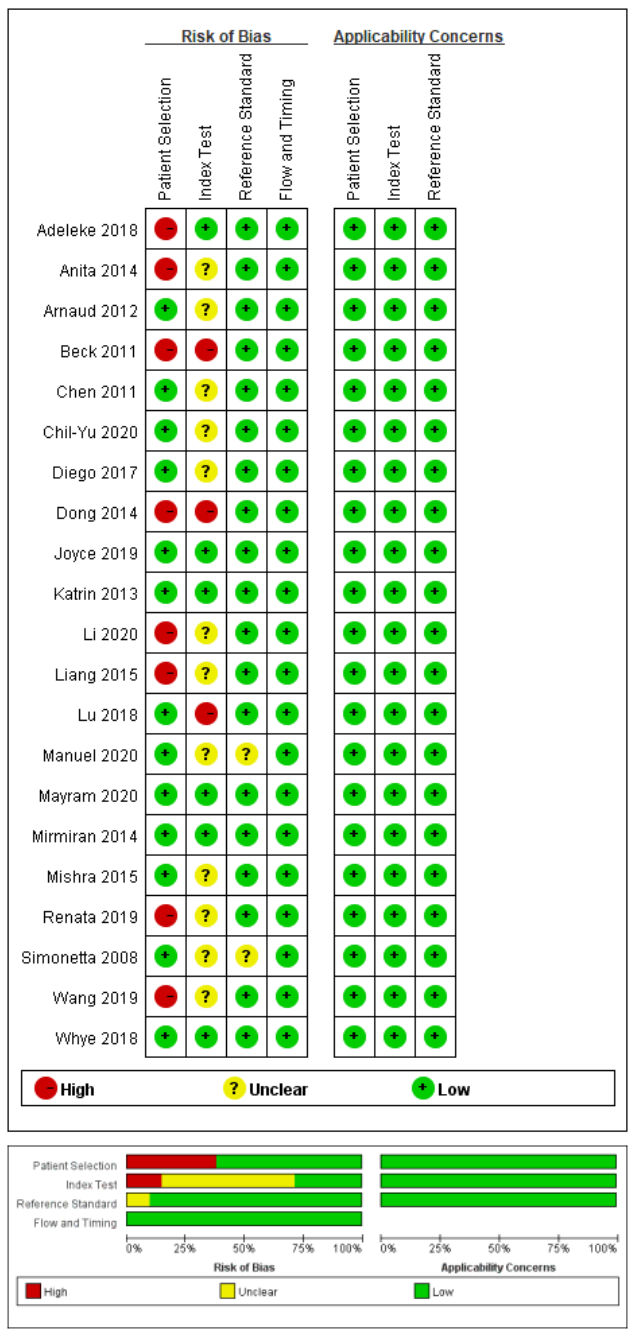

\section{Figure 2}

Methodological evaluation according to QUADAS-2 of included studies.
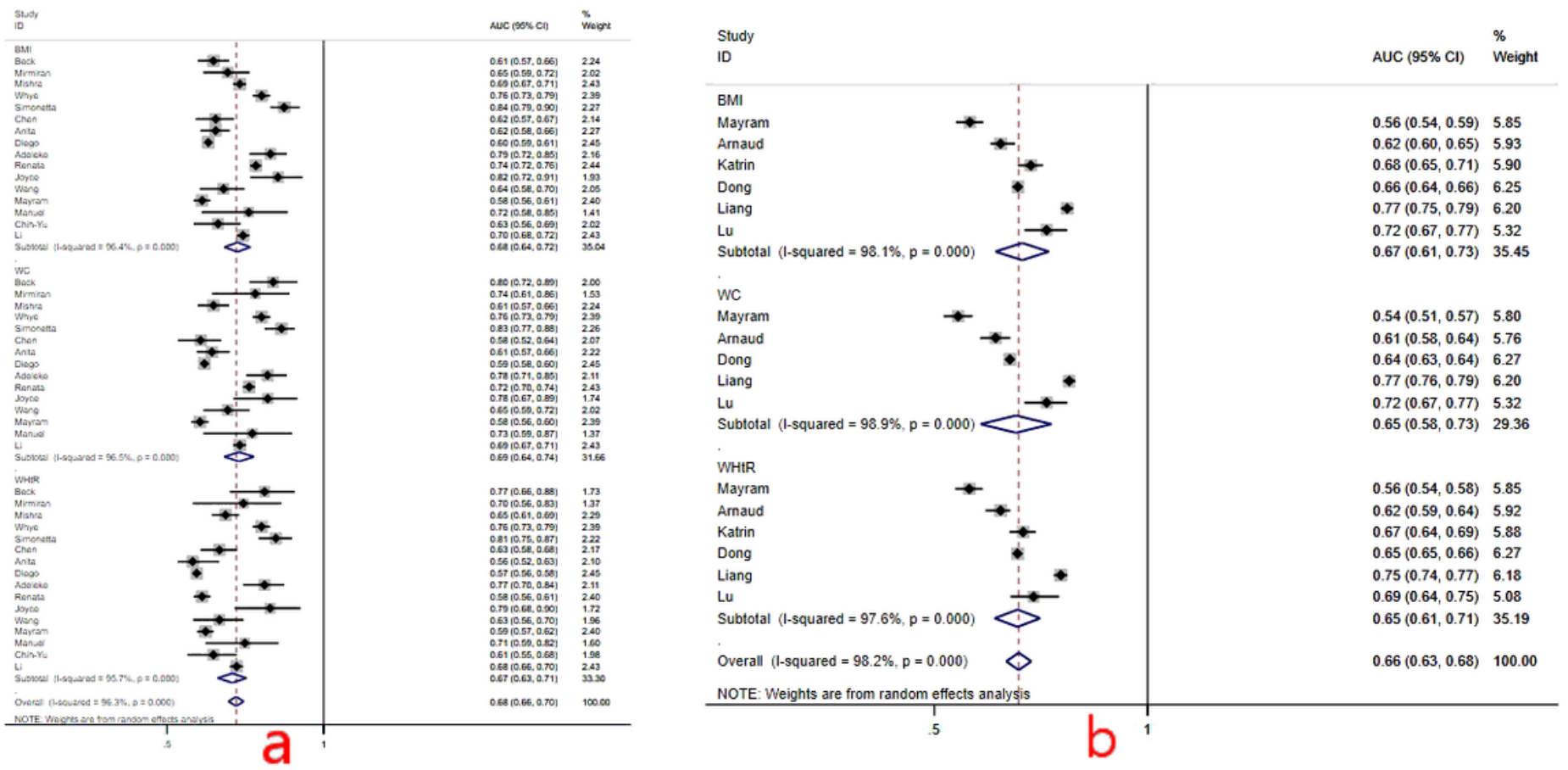
Figure 3

The pooled AUC values with $95 \% \mathrm{Cl}$ for hypertension and elevated BP in boys.

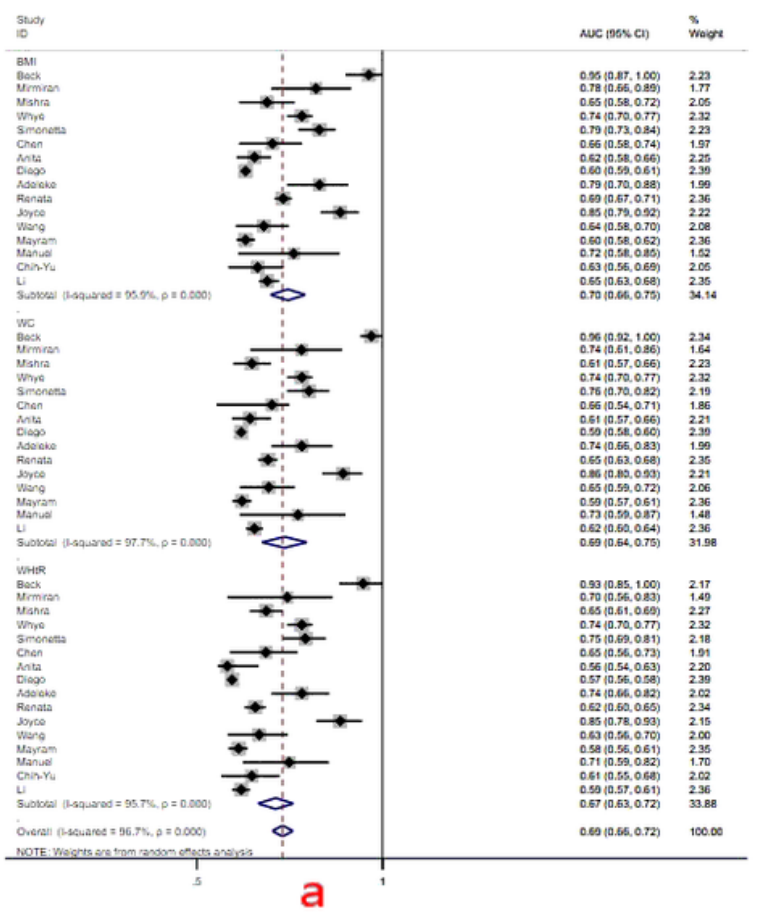

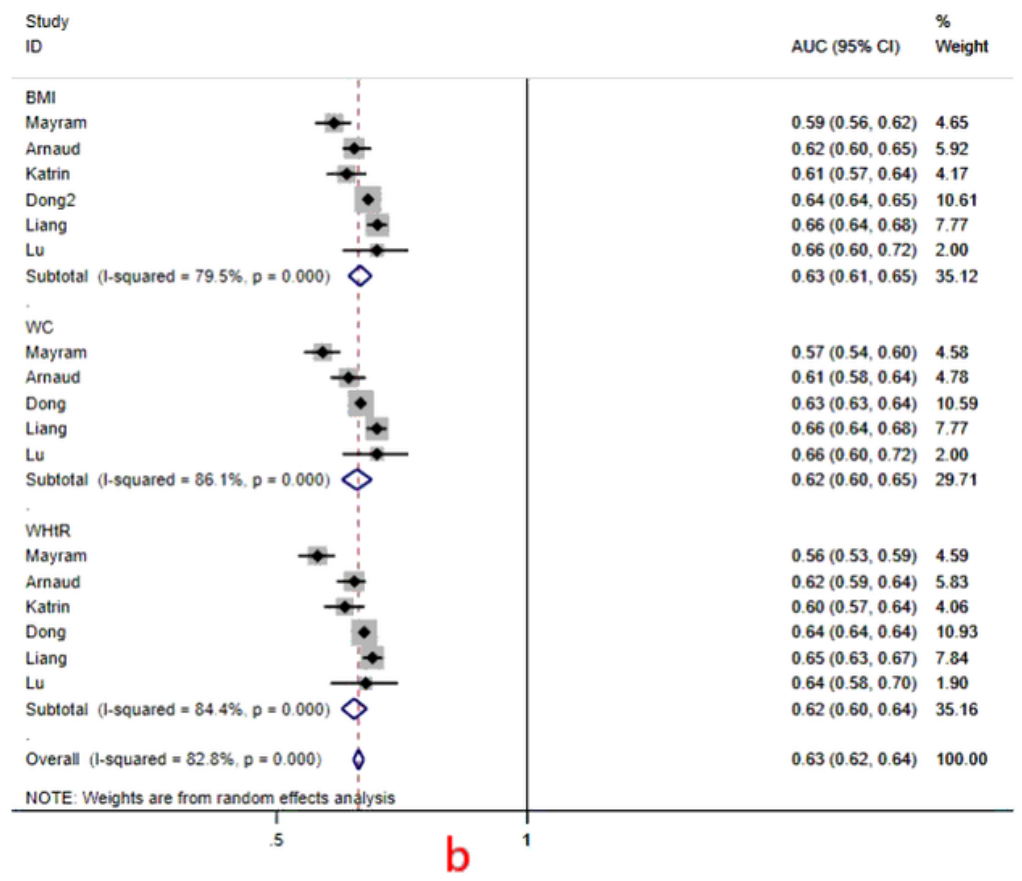

\section{Figure 4}

The pooled AUC values with $95 \% \mathrm{Cl}$ for hypertension and elevated BP in girls. 